\title{
Lavrentiev Regularization for Linear Ill-Posed Problems under General Source Conditions
}

\author{
M. T. Nair and U. Tautenhahn
}

\begin{abstract}
In this paper we study the problem of identifying the solution $x^{\dagger}$ of linear ill-posed problems $A x=y$ with non-negative and self-adjoint operators $A$ on a Hilbert space $X$ where instead of exact data $y$ noisy data $y^{\delta} \in X$ are given satisfying $\left\|y-y^{\delta}\right\| \leq \delta$ with known noise level $\delta$. Regularized approximations $x_{\alpha}^{\delta}$ are obtained by the method of Lavrentiev regularization, that is, $x_{\alpha}^{\delta}$ is the solution of the singularly perturbed operator equation $A x+\alpha x=y^{\delta}$, and the regularization parameter $\alpha$ is chosen either a priori or a posteriori by the rule of Raus. Assuming the unknown solution belongs to some general source set $M$ we prove that the regularized approximations provide order optimal error bounds on the set $M$. Our results cover the special case of finitely smoothing operators $A$ and extend recent results for infinitely smoothing operators. In addition, we generalize our results to the method of iterated Lavrentiev regularization of order $m$ and discuss a special ill-posed problem arising in inverse heat conduction.

Keywords: Ill-posed problems, inverse problems, Lavrentiev regularization, singular perturbation, a priori parameter choice, a posteriori rules, order optimal error bounds, general source conditions
\end{abstract}

AMS subject classification: 65J20, 65M30

\section{Introduction}

Ill-posed problems have important applications in science and engineering (see, e.g., $[2,9,13,14,29])$. In this paper we are interested in the solution $x^{\dagger} \in X$ of linear ill-posed problems

$$
A x=y \quad(y \in R(A))
$$

M. Thamban Nair: Indian Inst. Techn. Madras, Dept. Math., Chennai 600036 , India; e-mail mtnair@iitm.ac.in

U. Tautenhahn: Univ. Appl. Sci. Zittau/Görlitz, Dept. Math., P.O. Box 1454, D-02754 Zittau; e-mail u.tautenhahn@hs-zigr.de

ISSN 0232-2064 / \$2.50 C Heldermann Verlag Berlin 
where $A \in \mathcal{L}(X)$ is a non-negative, self-adjoint and injective operator with non-closed range $R(A)$, and $X$ is a Hilbert space. Here, $\mathcal{L}(X)$ denotes the space of all bounded linear operators from $X$ into itself. Throughout this paper we assume that $y^{\delta} \in X$ are the available noisy data with

$$
\left\|y-y^{\delta}\right\| \leq \delta
$$

and known noise level $\delta$.

The numerical treatment of ill-posed problems in which the solution $x^{\dagger}$ of problem (1.1) does not depend continuously on the data $y^{\delta}$ requires the application of special regularization methods. In the method of Tikhonov regularization (see $[2,9,14,29]$ ) a regularized approximation $x_{\alpha}^{\delta}$ of problem (1.1), (1.2) is defined as the solution of the minimization problem

$$
\min _{x \in X} J_{\alpha}(x) ; \quad J_{\alpha}(x)=\left\|A x-y^{\delta}\right\|^{2}+\alpha\|x\|^{2}
$$

depending on a positive regularization parameter $\alpha>0$.

In the case of non-negative and self-adjoint operators $A$ the least squares minimization in problem (1.3), equivalently

$$
A^{*} A x+\alpha x=A^{*} y^{\delta}
$$

can be replaced by the simpler regularized equation

$$
A x+\alpha x=y^{\delta}
$$

The method of computing the regularized approximation $x_{\alpha}^{\delta}$ by solving (1.5) is called method of Lavrentiev regularization (see [13, 24, 28, 30]) or, sometimes, simplified regularization (see [3 - 8]) or method of singular perturbation. The main aim of this paper is to extend some results from [21] for method (1.4) to the more simpler method (1.5).

The paper is organized as follows. In Section 2 we discuss some facts on optimality and order optimality of regularization methods for the approximate solution of problem (1.1) with data $y^{\delta} \in X$ satisfying condition (1.2). In Sections 3 and 4 we prove that the method of Lavrentiev regularization is order optimal on some general source set $M$ provided the regularization parameter is chosen either a priori (Section 3) or a posteriori by the rule of Raus (Section 4). In Section 5 we generalize our results to the method of iterated Lavrentiev regularization of order $m$ and in Section 6 we discuss a special ill-posed problem arising in inverse heat conduction and illustrate the assumptions required in the foregoing sections. 


\section{Optimality and order optimality}

For the stable approximate solution of problem (1.1) some regularization technique has to be applied, which provides regularized approximations $x_{\alpha}^{\delta}=$ $R_{\alpha}^{\delta} y^{\delta}$ with property $x_{\alpha}^{\delta} \rightarrow x^{\dagger}$ as $\delta \rightarrow 0$ where the regularization parameter $\alpha=\alpha\left(\delta, y^{\delta}\right)$ has to be chosen properly. Hence, regularized approximations $x_{\alpha}^{\delta}$ depend continuously on the data. However, the convergence of $x_{\alpha}^{\delta}$ to $x^{\dagger}$ can be arbitrarily slow without assuming additional quantitative a priori restrictions on the unknown solution $x^{\dagger}$, which is typical for ill-posed problems (see [25]).

In order to guarantee certain convergence rates for $\left\|x_{\alpha}^{\delta}-x^{\dagger}\right\|$, the set of solutions of problem (1.1) has to be restricted to certain source sets. Typically, for operator equations (1.1) with finitely smoothing operators $A$, source conditions of the type $x^{\dagger} \in M_{p, E}$ with

$$
M_{p, E}=\left\{x \in X \mid x=A^{p} v,\|v\| \leq E\right\} \quad(p>0)
$$

are exploited (see $[2,14,27,30]$ ). For infinitely smoothing operators $A$, source conditions of type (2.1) are generally too restrictive. In this case it is natural to assume that $x^{\dagger} \in M_{p, E}^{\log }$ with

$$
M_{p, E}^{\log }=\left\{x \in X \mid x=\ln ^{-p} A^{-1} v,\|v\| \leq E\right\} \quad(p>0)
$$

where the representation $x=\ln ^{-p} A^{-1} v$ in (2.2) has to be understood in the sense $x=\varphi(A) v$ with $\varphi(\lambda)=\left[\ln \frac{1}{\lambda}\right]^{-p}$, (see $\left.[10,15,22,27]\right)$. For the notation of finitely and infinitely smoothing forward operators see, e.g., [15]. In this paper we are interested in order optimality results under general source conditions $x^{\dagger} \in M_{\varphi, E}$ with $M_{\varphi, E}$ given by

$$
M_{\varphi, E}=\{x \in X \mid x=\varphi(A) v,\|v\| \leq E\}
$$

and source functions $\varphi$ satisfying

Assumption A1. $\varphi:(0, a] \rightarrow(0, \infty)$ with $\|A\| \leq a$ is continuous and possesses the following properties:

(i) $\varphi$ is strictly monotonically increasing on $(0, a]$ with $\lim _{\lambda \rightarrow 0} \varphi(\lambda)=0$.

(ii) The function $g:\left(0, \varphi^{2}(a)\right] \rightarrow\left(0, a^{2} \varphi^{2}(a)\right]$, implicitly defined by $g\left(\varphi^{2}(\lambda)\right)=\lambda^{2} \varphi^{2}(\lambda)$, is convex.

In $(2.3)$, the operator function $\varphi$ is defined via spectral representation

$$
\varphi(A)=\int_{0}^{a} \varphi(\lambda) d E_{\lambda} \quad(\|A\| \leq a)
$$


where $A=\int_{0}^{a} \lambda d E_{\lambda}$ is the spectral representation and $\left\{E_{\lambda}\right\}_{0 \leq \lambda \leq a}$ the spectral family of $A$ (see [20]). We may observe that, since $A$ is assumed to be selfadjoint and non-negative, $\sigma(A) \subseteq[0, a]$, where $\sigma(A)$ denotes the spectrum of the operator $A$.

Any operator $R: X \rightarrow X$ can be considered as a special method for solving problem (1.1). The approximate solution to (1.1) is then given by $R y^{\delta}$. Let us consider the worst case error $\Delta(\delta, R)$ for identifying the solution $x^{\dagger}$ of problem (1.1) from $y^{\delta} \in X$ under the assumptions $\left\|y-y^{\delta}\right\| \leq \delta$ and $x^{\dagger} \in M_{\varphi, E}$ which is defined by

$$
\Delta(\delta, R)=\sup \left\{\left\|R y^{\delta}-x^{\dagger}\right\| \mid x^{\dagger} \in M_{\varphi, E}, y^{\delta} \in X,\left\|y-y^{\delta}\right\| \leq \delta\right\} .
$$

This worst case error characterizes the maximal error of the method $R$ if the solution $x^{\dagger}$ of problem (1.1) varies in the set $M_{\varphi, E}$. An optimal method $R_{o p t}$ is characterized by $\Delta\left(\delta, R_{\text {opt }}\right)=\inf _{R} \Delta(\delta, R)$. It can easily be realized that

$$
\inf _{R} \Delta(\delta, R) \geq \omega\left(\delta, M_{\varphi, E}\right)
$$

with

$$
\omega\left(\delta, M_{\varphi, E}\right)=\sup \left\{\|x\| \mid x \in M_{\varphi, E},\|A x\| \leq \delta\right\} .
$$

For estimating the modulus of continuity $\omega\left(\delta, M_{\varphi, E}\right)$ of the inverse operator $A^{-1}$ on the source set $M_{\varphi, E}$, we introduce the function $\rho:(0, \varphi(a)] \rightarrow$ $(0, a \varphi(a)]$ defined implicitly by $\rho(\varphi(\lambda))=\lambda \varphi(\lambda)$, or explicitly by

$$
\rho(\lambda)=\lambda \varphi^{-1}(\lambda) \text {. }
$$

Theorem 2.1. Let $M_{\varphi, E}$ be given by (2.3) and let Assumption A1 be satisfied. If $\delta$ is sufficiently small such that $\frac{\delta}{E} \leq a \varphi(a)$, then

$$
\omega\left(\delta, M_{\varphi, E}\right) \leq E \rho^{-1}\left(\frac{\delta}{E}\right)
$$

with $\rho$ given by (2.5). If $\frac{\delta}{E} \in \sigma(A \varphi(A))$, then there holds equality in (2.6).

Proof. From (2.3) and (2.4) we have

$$
\omega\left(\delta, M_{\varphi, E}\right)=\sup \{\|\varphi(A) v\| \mid\|A \varphi(A) v\| \leq \delta,\|v\| \leq E\} .
$$

Substituting $\varphi(A) v=E w$ provides

$$
\omega\left(\delta, M_{\varphi, E}\right)=E \sup \left\{\|w\| \mid\|A w\| \leq \frac{\delta}{E},\left\|[\varphi(A)]^{-1} w\right\| \leq 1\right\} .
$$


Now choose $w$ in the range of $\varphi(A)$ such that the side conditions $\|A w\| \leq \frac{\delta}{E}$ and $\|\left[(\varphi(A)]^{-1} w \| \leq 1\right.$ of (2.8) are satisfied. Since the function $g$ of Assumption A1(ii) is convex, by exploiting Jensen's inequality we obtain

$$
\begin{aligned}
g\left(\frac{\|w\|^{2}}{\left\|[\varphi(A)]^{-1} w\right\|^{2}}\right) & =g\left(\frac{\int_{0}^{a} \varphi^{2}(\lambda)[\varphi(\lambda)]^{-2} d\left\|E_{\lambda} w\right\|^{2}}{\int_{0}^{a}[\varphi(\lambda)]^{-2} d\left\|E_{\lambda} w\right\|^{2}}\right) \\
& \leq \frac{\int_{0}^{a} g\left(\varphi^{2}(\lambda)\right)[\varphi(\lambda)]^{-2} d\left\|E_{\lambda} w\right\|^{2}}{\int_{0}^{a}[\varphi(\lambda)]^{-2} d\left\|E_{\lambda} w\right\|^{2}} \\
& =\frac{\int_{0}^{a} \lambda^{2} d\left\|E_{\lambda} w\right\|^{2}}{\int_{0}^{a}[\varphi(\lambda)]^{-2} d\left\|E_{\lambda} w\right\|^{2}} \\
& =\frac{\|A w\|^{2}}{\left\|[\varphi(A)]^{-1} w\right\|^{2}} .
\end{aligned}
$$

We exploit the side condition $\left\|[\varphi(A)]^{-1} w\right\| \leq 1$, i.e. $\|w\| \leq \frac{\|w\|}{\left\|[\varphi(A)]^{-1} w\right\|}$, take into consideration that the function $g$ implicitly defined in Assumption A1(ii) possesses the explicit form

$$
g(\lambda)=\lambda\left[\varphi^{-1}(\sqrt{\lambda})\right]^{2},
$$

exploit the monotonicity of $\varphi^{-1}$ as well as inequality (2.9) and obtain

$$
\begin{aligned}
{\left[\varphi^{-1}(\|w\|)\right]^{2} } & \leq\left[\varphi^{-1}\left(\frac{\|w\|}{\left\|[\varphi(A)]^{-1} w\right\|}\right)\right]^{2} \\
& =\frac{\left\|[\varphi(A)]^{-1} w\right\|^{2}}{\|w\|^{2}} g\left(\frac{\|w\|^{2}}{\left\|[\varphi(A)]^{-1} w\right\|^{2}}\right) \\
& \leq \frac{\|A w\|^{2}}{\|w\|^{2}} .
\end{aligned}
$$

This inequality attains the form $\rho(\|w\|) \leq\|A w\|$, giving $\|w\| \leq \rho^{-1}(\|A w\|)$, where $\rho$ is defined by (2.5). Due to the monotonicity of $\rho^{-1}$ and the assumption $\frac{\delta}{E} \leq a \varphi(a)$ we obtain $\|w\| \leq \rho^{-1}(\|A w\|) \leq \rho^{-1}\left(\frac{\delta}{E}\right)$. From this estimate and (2.8) we obtain (2.6).

In the second part we prove that in (2.6) equality holds provided $\frac{\delta}{E} \in$ $\sigma(A \varphi(A))$. Assume that $\frac{\delta}{E}$ is an eigenvalue of the operator $A \varphi(A)$ and $v_{0}$ is a corresponding eigenvector with $\left\|v_{0}\right\|=E$. Then $A \varphi(A) v_{0}=\frac{\delta}{E} v_{0}$, consequently, $\left\|A \varphi(A) v_{0}\right\|=\delta$. Hence, in view of (2.7) we conclude that $\omega\left(\delta, M_{\varphi, E}\right) \geq\left\|\varphi(A) v_{0}\right\|$. From $\rho(\varphi(A)) v_{0}=A \varphi(A) v_{0}=\frac{\delta}{E} v_{0}$ we obtain $\varphi(A) v_{0}=\rho^{-1}\left(\frac{\delta}{E}\right) v_{0}$, consequently, $\omega\left(\delta, M_{\varphi, E}\right) \geq E \rho^{-1}\left(\frac{\delta}{E}\right)$. Hence, due to (2.6) we have

$$
\omega\left(\delta, M_{\varphi, E}\right)=E \rho^{-1}\left(\frac{\delta}{E}\right) .
$$


If $\frac{\delta}{E} \in \sigma(A \varphi(A))$ is not an eigenvalue, then $\frac{\delta}{E}$ belongs to the approximate eigenspectrum of $A \varphi(A)$ as $A \varphi(A)$ is self-adjoint (cf. [20]), and in that case, the proof of (2.12) follows with small modifications

For the proof of Theorem 2.1 in the case of compact operators $A$ see [11]. Our proof is based on the proof of Theorem 2.1 in [27] and it is more general since the operator $A$ is not necessarily compact. Note that estimate (2.6) can also be given in terms of the function $g$ defined in Assumption A1(ii) and possesses the equivalent form $\omega\left(\delta, M_{\varphi, E}\right) \leq E \sqrt{g^{-1}\left(\frac{\delta^{2}}{E^{2}}\right)}$. A further equivalent variant of (2.6) which is along the lines of [16] uses the function $\Theta(\lambda)=\lambda \varphi(\lambda)$ and has the form $\omega\left(\delta, M_{\varphi, E}\right) \leq E \varphi\left(\Theta^{-1}\left(\frac{\delta}{E}\right)\right)$.

Due to Theorem 2.1 the following definition makes sense.

Definition 2.2. Let Assumption A1 be satisfied and $\rho$ be given by (2.5). Then, any regularization method $R_{\alpha}^{\delta}$, or any regularized approximation $x_{\alpha}^{\delta}=$ $R_{\alpha}^{\delta} y^{\delta}$ for problem (1.1), (1.2) is called

(i) optimal on the set $M_{\varphi, E}$ if $\left\|x_{\alpha}^{\delta}-x^{\dagger}\right\| \leq E \rho^{-1}\left(\frac{\delta}{E}\right)$

(ii) order optimal on the set $M_{\varphi, E}$ if $\left\|x_{\alpha}^{\delta}-x^{\dagger}\right\| \leq c E \rho^{-1}\left(\frac{\delta}{E}\right)$ with $c \geq 1$.

\section{A priori parameter choice}

In this section we prove that for proper a priori chosen regularization parameter $\alpha$ the method of Lavrentiev regularization

$$
x_{\alpha}^{\delta}=(A+\alpha I)^{-1} y^{\delta}
$$

yields order optimal error bounds on the set $M_{\varphi, E}$ provided $\varphi$ is concave. In our first proposition of this section we estimate the regularization error $\left\|x_{\alpha}-x^{\dagger}\right\|$ where $x_{\alpha}$ is the regularized approximation with exact data, that is,

$$
x_{\alpha}=(A+\alpha I)^{-1} y \text {. }
$$

Proposition 3.1. Let $M_{\varphi, E}$ be given by (2.3), $x^{\dagger} \in M_{\varphi, E}$, Assumption $\mathrm{A} 1$ be satisfied and let $\rho$ be given by (2.5). Let $x_{\alpha}$ be the regularized approximation defined in (3.2) and let $\alpha$ be chosen a priori by

$$
\alpha=\varphi^{-1}\left(\rho^{-1}\left(\frac{\delta}{E}\right)\right)
$$

If the function $\varphi$ is concave, then

$$
\left\|x_{\alpha}-x^{\dagger}\right\| \leq E \rho^{-1}\left(\frac{\delta}{E}\right)
$$


Proof. Since $\lim _{\lambda \rightarrow 0} \varphi(\lambda)=0$ and since $\varphi$ is concave we have $t \varphi(\lambda) \leq$ $\varphi(t \lambda)$ for $t \in[0,1]$. Choosing $t=\frac{\alpha}{\lambda+\alpha}$ and exploiting the monotonicity of $\varphi$ provides

$$
\frac{\alpha}{\lambda+\alpha} \varphi(\lambda) \leq \varphi\left(\frac{\alpha \lambda}{\lambda+\alpha}\right) \leq \varphi(\alpha) .
$$

Let us use the notation

$$
B_{\alpha}=I-A(A+\alpha I)^{-1}=\alpha(A+\alpha I)^{-1} .
$$

Then, the regularization error can be expressed by $\left\|x_{\alpha}-x^{\dagger}\right\|=\left\|B_{\alpha} x^{\dagger}\right\|$. Exploiting the source condition $x^{\dagger} \in M_{\varphi, E}$ with $x^{\dagger}=\varphi(A) v$ and applying (3.5) provides

$$
\left\|x_{\alpha}-x^{\dagger}\right\|=\left\|B_{\alpha} x^{\dagger}\right\| \leq\left\|B_{\alpha} \varphi(A) v\right\| \leq E \sup _{\lambda \geq 0}\left|\frac{\alpha}{\lambda+\alpha} \varphi(\lambda)\right| \leq E \varphi(\alpha) .
$$

For the regularization parameter $\alpha$ chosen by (3.3) there holds $\varphi(\alpha)=\rho^{-1}\left(\frac{\delta}{E}\right)$. Consequently, (3.4) follows from (3.6)

Our next theorem provides an order optimal error bound for $\left\|x_{\alpha}^{\delta}-x^{\dagger}\right\|$ provided the regularization parameter $\alpha$ is chosen a priori by (3.3).

Theorem 3.2. Let $M_{\varphi, E}$ be given by (2.3), $x^{\dagger} \in M_{\varphi, E}$, Assumption $\mathrm{A} 1$ be satisfied and let $\rho$ be given by (2.5). Let $x_{\alpha}^{\delta}$ be the regularized approximation defined in (3.1) and let $\alpha$ be chosen a priori by (3.3). If the function $\varphi$ is concave, then

$$
\left\|x_{\alpha}^{\delta}-x^{\dagger}\right\| \leq 2 E \rho^{-1}\left(\frac{\delta}{E}\right) .
$$

Proof. Due to $\rho(\lambda)=\lambda \varphi^{-1}(\lambda)$ we obtain for $\lambda=\rho^{-1}\left(\frac{\delta}{E}\right)$ the equation

$$
\frac{\delta}{E}=\rho^{-1}\left(\frac{\delta}{E}\right) \varphi^{-1}\left(\rho^{-1}\left(\frac{\delta}{E}\right)\right) .
$$

Hence, for $\alpha$ chosen by (3.3) we obtain $\frac{\delta}{E}=\alpha \rho^{-1}\left(\frac{\delta}{E}\right)$ or, equivalently,

$$
\frac{\delta}{\alpha}=E \rho^{-1}\left(\frac{\delta}{E}\right)
$$

Let $x_{\alpha}$ be given by (3.2). Then, by (3.8),

$$
\left\|x_{\alpha}^{\delta}-x_{\alpha}\right\|=\left\|(A+\alpha I)^{-1}\left(y^{\delta}-y\right)\right\| \leq \frac{\delta}{\alpha}=E \rho^{-1}\left(\frac{\delta}{E}\right) .
$$

Now (3.7) follows from (3.4), (3.9) and the triangle inequality 
Let us discuss two special cases that fit into the framework of Theorem 3.2. In our first special case we consider operator equations with finitely smoothing operators $A$ and assume that the function $\varphi$ of set (2.3) has the form $\varphi(\lambda)=\lambda^{p}$ with $p>0$. It can be easily seen that this function satisfies the requirements of Assumption A1. Since $\varphi^{-1}(\lambda)=\lambda^{\frac{1}{p}}$ we obtain $\rho(\lambda):=\lambda \varphi^{-1}(\lambda)=\lambda^{\frac{p+1}{p}}$, consequently, $\rho^{-1}(\lambda)=\lambda^{\frac{p}{p+1}}$, which provides $\varphi^{-1}\left(\rho^{-1}(\lambda)\right)=\lambda^{\frac{1}{p+1}}$. Hence, the regularization parameter (3.3) attains the form

$$
\alpha=\left(\frac{\delta}{E}\right)^{\frac{1}{p+1}}
$$

Since $\varphi(\lambda)=\lambda^{p}$ is concave for $p \leq 1$ we obtain the following result from Theorem 3.2.

Corollary 3.3. Let $M_{p, E}$ be given by (2.1), $x^{\dagger} \in M_{p, E}$ and let $x_{\alpha}^{\delta}$ be the regularized approximation defined in (3.1). Let $\alpha$ be chosen a priori by (3.10). If $0<p \leq 1$, then $x_{\alpha}^{\delta}$ is order optimal on the set $M_{p, E}$, and

$$
\left\|x_{\alpha}^{\delta}-x^{\dagger}\right\| \leq 2 E^{\frac{1}{p+1}} \delta^{\frac{p}{p+1}} .
$$

In our second special case we consider operator equations with infinitely smoothing operators $A$ and assume that the function $\varphi$ of set (2.3) has the form $\varphi(\lambda)=\left[\ln \frac{1}{\lambda}\right]^{-p}$ with $p>0$. We observe that Assumption A1 is satisfied for all $p>0$ provided $\|A\| \leq e^{-\frac{1}{2}}$. Since $\varphi^{-1}(\lambda)=\mathrm{e}^{-1 / \lambda^{\frac{1}{p}}}$ we obtain $\rho(\lambda):=$ $\lambda \varphi^{-1}(\lambda)=\lambda \mathrm{e}^{-1 / \lambda^{\frac{1}{p}}}$, consequently,

$$
\rho^{-1}(\lambda)=\left[\ln \frac{1}{\lambda}\right]^{-p}(1+o(1)) \quad(\lambda \rightarrow 0)
$$

(see, e.g., [15]). Consequently, the regularization parameter (3.3) attains the form

$$
\alpha=\frac{\delta}{E}\left[\ln \frac{E}{\delta}\right]^{p}(1+o(1)) \quad(\delta \rightarrow 0) .
$$

Since $\varphi$ is concave for $\lambda \leq \mathrm{e}^{-(p+1)}$ we obtain the following result from Theorem 3.2 .

Corollary 3.4. Let $M_{p, E}^{\log }$ be given by $(2.2), x^{\dagger} \in M_{p, E}^{\log }, x_{\alpha}^{\delta}$ be the regularized approximation (3.1) and let $\alpha$ be chosen a priori by (3.13). If $\|A\| \leq$ $\mathrm{e}^{-(p+1)}$, then $x_{\alpha}^{\delta}$ is order optimal on the set $M_{p, E}^{\log }$ and

$$
\left\|x_{\alpha}^{\delta}-x^{\dagger}\right\| \leq 2 E\left[\ln \frac{E}{\delta}\right]^{-p}(1+o(1)) \quad(\delta \rightarrow 0) .
$$




\section{A posteriori parameter choice}

In Section 3 we have proved that the method of Lavrentiev regularization provides order optimal error bounds (3.7) on the general set $M_{\varphi, E}$ given by (2.3) provided the regularization parameter $\alpha$ is chosen a priori according to formula (3.3). Unfortunately, this a priori parameter choice requires the knowledge of the function $\varphi$, which is generally unknown. One prominent $a$ posteriori rule for choosing $\alpha$ which does not require to know the function $\varphi$ is Morozov's discrepancy principle (see [17, 18]) in which $\alpha$ is chosen as the solution of the nonlinear scalar equation $\left\|A x_{\alpha}^{\delta}-y^{\delta}\right\|=C \delta$ with some constant $C \geq 1$. Although Morozovs's discrepancy principle works well for the method of Tikhonov regularization (1.3) (see, for example, [21, 27]), it appears to be divergent for the method of Lavrentiev regularization (see $[7,30]$ ).

A convergent a posteriori rule for the method of Lavrentiev regularization has been studied in [4]. In this rule the regularization parameter $\alpha$ is chosen as the solution of the nonlinear equation

$$
\alpha^{t}\left\|A x_{\alpha}^{\delta}-y^{\delta}\right\|=\delta^{s}
$$

For this rule order optimality on the source set $M_{p, E}$ given in (2.1) has been established for the range $p \in(0,1]$ provided the non-negative numbers $s, t, p$ are related by $s=\frac{t+1}{p+1}$. Hence, in order to guarantee order optimality for this rule, the knowledge of the number $p$ in the source set (2.1) is required.

In this section we discuss the rule of Raus (see [3, 8, 24]) for choosing the regularization parameter. This a posteriori rule does not require to know the number $p$ in the source set (2.1), and more generally, does not require to know the function $\varphi$ which characterizes the set $M_{\varphi, E}$ given by (2.3). This rule reads as follows:

Rule of Raus. For given constant $C>1$, choose $\alpha$ as the solution of the equation

$$
d(\alpha):=\left\|B_{\alpha}\left(A x_{\alpha}^{\delta}-y^{\delta}\right)\right\|=C \delta \quad \text { with } \quad B_{\alpha}=\alpha(A+\alpha I)^{-1} .
$$

The nonlinear scalar equation (4.1) possesses a unique solution $\alpha=\alpha_{R}$ provided $\left\|y^{\delta}\right\|>C \delta$ (see $\left.[3,24,28]\right)$. We prove in this section that for concave functions $\varphi$ the method of Lavrentiev regularization combined with rule (4.1) is order optimal on the source set $M_{\varphi, E}$ given by (2.3).

In our first proposition we estimate the regularization error $\left\|x_{\alpha}-x^{\dagger}\right\|$ where $x_{\alpha}$ is the regularized approximation with exact data, that is, $x_{\alpha}$ is given by (3.2). 
Proposition 4.1. Let $x^{\dagger} \in M_{\varphi, E}$ with $M_{\varphi, E}$ given by (2.3), let Assumption $\mathrm{A} 1$ be satisfied and let $\rho$ be given by (2.5). Let $x_{\alpha}$ the regularized approximation defined in (3.2) and let $\alpha$ be chosen by rule (4.1). If the function $\varphi$ is concave, then

$$
\left\|x_{\alpha}-x^{\dagger}\right\| \leq(C+1) E \rho^{-1}\left(\frac{\delta}{E}\right) .
$$

Proof. Let us use the notations

$$
B_{\alpha}=I-A(A+\alpha I)^{-1}=\alpha(A+\alpha I)^{-1} \quad \text { and } \quad r_{\alpha}(\lambda)=\frac{\alpha}{\lambda+\alpha} .
$$

Since $\varphi$ is a concave function with $\lim _{\lambda \rightarrow 0} \varphi(\lambda)=0$ we have $t \varphi(\lambda) \leq \varphi(t \lambda)$ for $t \in[0,1]$, or equivalently, $\left[\varphi^{-1}(t \varphi(\lambda))\right]^{2} \leq t^{2} \lambda^{2}$. We multiply by $t^{2} \varphi^{2}(\lambda)$ and obtain

$$
t^{2} \varphi^{2}(\lambda)\left[\varphi^{-1}(t \varphi(\lambda))\right]^{2} \leq t^{4} \lambda^{2} \varphi^{2}(\lambda) \quad(t \in[0,1]) .
$$

Recall that the function $g$ from Assumption A1(ii) possesses the explicit form (2.10). Hence, (4.4) attains the form $g\left(t^{2} \varphi^{2}(\lambda)\right) \leq t^{4} \lambda^{2} \varphi^{2}(\lambda)$. Choosing $t=r_{\alpha}(\lambda)$ yields

$$
g\left(r_{\alpha}^{2}(\lambda) \varphi^{2}(\lambda)\right) \leq \lambda^{2} r_{\alpha}^{4}(\lambda) \varphi^{2}(\lambda) .
$$

Let $\alpha$ be the regularization parameter chosen by rule (4.1). Since $d(\alpha)=$ $\left\|B_{\alpha}^{2} y^{\delta}\right\|$ we obtain

$$
\left\|B_{\alpha}^{2} y\right\| \leq\left\|B_{\alpha}^{2} y^{\delta}\right\|+\left\|B_{\alpha}^{2}\left(y-y^{\delta}\right)\right\| \leq(C+1) \delta .
$$

Note that since $x^{\dagger} \in M_{\varphi, E}, x^{\dagger}=\varphi(A) v$ for some $v \in X$ with $\|v\| \leq E$, so that by (4.3) we have $B_{\alpha} x^{\dagger}=r_{\alpha}(A) \varphi(A) v$. Now we use Assumption A1, (4.5), (4.6) and obtain, by exploiting Jensen's inequality, that

$$
\begin{aligned}
g\left(\frac{\left\|B_{\alpha} x^{\dagger}\right\|^{2}}{\|v\|^{2}}\right) & =g\left(\frac{\int_{0}^{a} r_{\alpha}^{2}(\lambda) \varphi^{2}(\lambda) d\left\|E_{\lambda} v\right\|^{2}}{\int_{0}^{a} d\left\|E_{\lambda} v\right\|^{2}}\right) \\
& \leq \frac{\int_{0}^{a} g\left(r_{\alpha}^{2}(\lambda) \varphi^{2}(\lambda)\right) d\left\|E_{\lambda} v\right\|^{2}}{\int_{0}^{a} d\left\|E_{\lambda} v\right\|^{2}} \\
& \leq \frac{\int_{0}^{a} \lambda^{2} r_{\alpha}^{4}(\lambda) \varphi^{2}(\lambda) d\left\|E_{\lambda} v\right\|^{2}}{\int_{0}^{a} d\left\|E_{\lambda} v\right\|^{2}} \\
& =\frac{\left\|B_{\alpha}^{2} A x^{\dagger}\right\|^{2}}{\|v\|^{2}} \\
& \leq \frac{(C+1)^{2} \delta^{2}}{\|v\|^{2}} .
\end{aligned}
$$


Using the monotonicity of $\varphi^{-1}$ as well as relations (2.10) and (4.7) we obtain

$$
\begin{aligned}
{\left[\varphi^{-1}\left(\frac{\left\|B_{\alpha} x^{\dagger}\right\|}{(C+1) E}\right)\right]^{2} } & \leq\left[\varphi^{-1}\left(\frac{\left\|B_{\alpha} x^{\dagger}\right\|}{\|v\|}\right)\right]^{2} \\
& =\frac{\|v\|^{2}}{\left\|B_{\alpha} x^{\dagger}\right\|^{2}} g\left(\frac{\left\|B_{\alpha} x^{\dagger}\right\|^{2}}{\|v\|^{2}}\right) \\
& \leq \frac{(C+1)^{2} \delta^{2}}{\left\|B_{\alpha} x^{\dagger}\right\|^{2}} .
\end{aligned}
$$

Due to the definition of $\rho$ according to $\rho(\lambda)=\lambda \varphi^{-1}(\lambda)$, the above estimate provides $\rho\left(\frac{\left\|B_{\alpha} x^{\dagger}\right\|}{(C+1) E}\right) \leq \frac{\delta}{E}$. From this estimate and the identity $\left\|x_{\alpha}-x^{\dagger}\right\|=$ $\left\|B_{\alpha} x^{\dagger}\right\|$ we obtain (4.2)

Our next proposition provides some estimate for the regularization parameter obtained by rule (4.1).

Proposition 4.2. Let $M_{\varphi, E}$ be given by (2.3), $x^{\dagger} \in M_{\varphi, E}$, and let Assumption A1 be satisfied. Let $\alpha$ be chosen by rule (4.1). If the function $\varphi$ is concave, then

$$
(C-1) \delta \leq E \alpha \varphi(\alpha) .
$$

Proof. Let us use notations (4.3). From rule (4.1) we obtain

$$
C \delta=\left\|B_{\alpha}^{2} y^{\delta}\right\| \leq \delta+\left\|B_{\alpha}^{2} y\right\| .
$$

From this estimate, the estimate $\left\|B_{\alpha} A\right\| \leq \alpha$ and (3.6) we obtain

$$
(C-1) \delta \leq\left\|B_{\alpha}^{2} y\right\|=\left\|B_{\alpha}^{2} A x^{\dagger}\right\| \leq \alpha\left\|B_{\alpha} x^{\dagger}\right\| \leq E \alpha \varphi(\alpha) .
$$

Hence, the proof is complete

Now we are ready to provide our main result of this section.

Theorem 4.3. Let $M_{\varphi, E}$ be given by $(2.3)$, $x^{\dagger} \in M_{\varphi, E}$, and let Assumption A1 be satisfied. Let $x_{\alpha}^{\delta}$ the regularized approximation defined in (3.1) and let $\alpha$ be chosen by rule (4.1). If the function $\varphi$ is concave, then $x_{\alpha}^{\delta}$ is order optimal on the set $M_{\varphi, E}$. In fact,

$$
\left\|x_{\alpha}^{\delta}-x^{\dagger}\right\| \leq c_{0} E \rho^{-1}\left(\frac{\delta}{E}\right)
$$

with $c_{0}=C+1+\frac{1}{C-1}$ for $1<C \leq 2$ and $c_{0}=C+2$ for $C \geq 2$.

Proof. From (4.8) and the monotonicity of $\varphi$ we conclude that

$$
\varphi^{-1}\left(\frac{(C-1) \delta}{E \alpha}\right) \leq \alpha .
$$


Since $\rho(\lambda)=\lambda \varphi^{-1}(\lambda)$ we obtain that $\rho\left(\frac{(C-1) \delta}{E \alpha}\right) \leq \frac{(C-1) \delta}{E}$ or, equivalently,

$$
\frac{(C-1) \delta}{E \alpha} \leq \rho^{-1}\left(\frac{(C-1) \delta}{E}\right)
$$

We exploit the monotonicity of $\rho^{-1}$ and obtain in the case $C \leq 2$ that

$$
\frac{\delta}{\alpha} \leq \frac{E}{C-1} \rho^{-1}\left(\frac{\delta}{E}\right) \quad \text { for } \quad 1<C \leq 2
$$

In the case $C \geq 2$ we use the monotonicity of $\varphi^{-1}$ and obtain from (4.10) the estimate $\varphi^{-1}\left(\frac{\delta}{E \alpha}\right) \leq \alpha$, and instead of (4.11) the estimate

$$
\frac{\delta}{\alpha} \leq E \rho^{-1}\left(\frac{\delta}{E}\right) \quad \text { for } C \geq 2
$$

From (3.1) and (3.2) we have $\left\|x_{\alpha}^{\delta}-x_{\alpha}\right\| \leq \frac{\delta}{\alpha}$, consequently, due to (4.11) and $(4.12)$,

$$
\left\|x_{\alpha}^{\delta}-x_{\alpha}\right\| \leq c E \rho^{-1}\left(\frac{\delta}{E}\right)
$$

with $c=\frac{1}{C-1}$ for $1<C \leq 2$ and $c=1$ for $C \geq 2$. Now the order optimal error bound (4.9) follows from (4.2) and (4.13)

As in Section 3, let us discuss two special cases that fit into the framework of Theorem 4.3. In our first special case we consider operator equations with finitely smoothing operators $A$ and assume that the function $\varphi$ of set (2.3) has the form $\varphi(\lambda)=\lambda^{p}$ with $p>0$. This function satisfies the requirements of Assumption A1, and $\rho^{-1}$ has the form $\rho^{-1}(\lambda)=\lambda^{\frac{p}{p+1}}$. Since $\varphi(\lambda)=\lambda^{p}$ is concave for $p \leq 1$ we obtain the following result from Theorem 4.3.

Corollary 4.4. Let $M_{p, E}$ given by (2.1), $x^{\dagger} \in M_{p, E}, x_{\alpha}^{\delta}$ be the regularized approximation (3.1) and let $\alpha$ be chosen by rule (4.1). If $0<p \leq 1$, then $x_{\alpha}^{\delta}$ is order optimal on the source set $M_{p, E}$. In fact,

$$
\left\|x_{\alpha}^{\delta}-x^{\dagger}\right\| \leq c_{0} E^{\frac{1}{p+1}} \delta^{\frac{p}{p+1}}
$$

with $c_{0}$ as in Theorem 4.3 .

In our second special case we consider as in Section 3 operator equations with infinitely smoothing operators $A$ and assume that the function $\varphi$ of set (2.3) has the form $\varphi(\lambda)=\left[\ln \frac{1}{\lambda}\right]^{-p}$ for $p>0$. This function satisfies the requirements of Assumption A1 provided $p>0$ and $\|A\| \leq \mathrm{e}^{-\frac{1}{2}}$, and $\rho^{-1}$ attains form (3.12). Since $\varphi$ is concave for $\lambda \leq \mathrm{e}^{-(p+1)}$ we obtain the following result from Theorem 4.3 . 
Corollary 4.5. Let $M_{p, E}^{\log }$ given by $(2.2), x^{\dagger} \in M_{p, E}^{\log }, x_{\alpha}^{\delta}$ be the regularized approximation defined in (3.1) and let $\alpha$ be chosen by rule (4.1). If $\|A\| \leq$ $e^{-(p+1)}$, then $x_{\alpha}^{\delta}$ is order optimal on the source set $M_{p, E}^{\log }$. In fact,

$$
\left\|x_{\alpha}^{\delta}-x^{\dagger}\right\| \leq c_{0} E\left[\ln \frac{E}{\delta}\right]^{-p}(1+o(1)) \quad(\delta \rightarrow 0)
$$

with $c_{0}$ as in Theorem 4.3 .

\section{Iterated Lavrentiev regularization}

In this section we are going to generalize our results of Sections 3 and 4 for the method of iterated Lavrentiev regularization of order $m$. Starting with $x_{\alpha, 0}^{\delta}=0$, in this method the regularized approximation $x_{\alpha}^{\delta}:=x_{\alpha, m}^{\delta}$ is defined recursively by solving the $m$ operator equations

$$
(A+\alpha I) x_{\alpha, k}^{\delta}=y^{\delta}+\alpha x_{\alpha, k-1}^{\delta} \quad(k=1, \ldots, m) .
$$

The advantage of this method over method (1.4) consists in the fact that order optimality results hold true for a larger class of source functions $\varphi$ of the source set (2.3), especially for such source functions for which $\varphi^{\frac{1}{m}}$ is concave.

In the case of exact data $y$, we define $x_{\alpha}:=x_{\alpha, m}$ recursively by solving the $m$ operator equations

$$
(A+\alpha I) x_{\alpha, k}=y+\alpha x_{\alpha, k-1} \quad(k=1, \ldots, m) .
$$

We may observe that $x_{\alpha}^{\delta}=g_{\alpha}(A) y^{\delta}$ and $x_{\alpha}=g_{\alpha}(A) y$ where

$$
g_{\alpha}(\lambda)=\frac{1}{\lambda}\left[1-\left(\frac{\alpha}{\lambda+\alpha}\right)^{m}\right] \quad(0<\lambda \leq\|A\|) .
$$

In the case of a priori parameter choice, in place of Theorem 3.2 we have the following result.

Theorem 5.1. Let $M_{\varphi, E}$ be given by (2.3), $x^{\dagger} \in M_{\varphi, E}$, Assumption A1 be satisfied and let $\rho$ be given by (2.5). Let $x_{\alpha}^{\delta}:=x_{\alpha, m}^{\delta}$ the regularized approximation defined in (5.1) and let $\alpha$ be chosen a priori by (3.3). If the function $\varphi^{\frac{1}{m}}$ is concave, then

$$
\left\|x_{\alpha}^{\delta}-x^{\dagger}\right\| \leq(1+m) E \rho^{-1}\left(\frac{\delta}{E}\right)
$$

Proof. Let us use the notations in (4.3). Due to the concavity of $\varphi^{\frac{1}{m}}$ we have $t^{m} \varphi(\lambda) \leq \varphi(t \lambda)$ for $0<t \leq 1$. We use this inequality with $t=r_{\alpha}(\lambda)$, 
exploit the representation $x^{\dagger}-x_{\alpha}=B_{\alpha}^{m} x^{\dagger}$ and obtain due to $x^{\dagger} \in M_{\varphi, E}$ and the monotonicity of $\varphi$ that

$$
\left\|x_{\alpha}-x^{\dagger}\right\| \leq E \sup _{\lambda \in(0, a]}\left|r_{\alpha}^{m}(\lambda) \varphi(\lambda)\right| \leq E \varphi\left(\lambda r_{\alpha}(\lambda)\right) \leq E \varphi(\alpha) .
$$

For estimating $\left\|x_{\alpha}^{\delta}-x_{\alpha}\right\|$ we observe that $x_{\alpha}^{\delta}-x_{\alpha}=g_{\alpha}(A)\left(y^{\delta}-y\right)$ with $g_{\alpha}$ as in (5.3). Since $\left|g_{\alpha}(\lambda)\right| \leq m \alpha$, it follows that

$$
\left\|x_{\alpha}^{\delta}-x_{\alpha}\right\| \leq m \frac{\delta}{\alpha}
$$

For $\alpha$ chosen according to (3.3) we have $\varphi(\alpha)=\rho^{-1}\left(\frac{\delta}{E}\right)$ and $\frac{\delta}{\alpha}=E \rho^{-1}\left(\frac{\delta}{E}\right)$ (compare (3.8)). Hence, the desired error bound (5.4) follows from (5.5) and (5.6)

In the case of a posteriori parameter choice, instead of Theorem 4.3 we have the following result.

Theorem 5.2. Let $M_{\varphi, E}$ be given by (2.3), $x^{\dagger} \in M_{\varphi, E}$, and let Assumption $\mathrm{A} 1$ be satisfied. Let $x_{\alpha}^{\delta}:=x_{\alpha, m}^{\delta}$ the regularized approximation defined via (5.1) and let $\alpha$ be chosen by rule (4.1). If the function $\varphi^{\frac{1}{m}}$ is concave, then $x_{\alpha}^{\delta}$ is order optimal on the set $M_{\varphi, E}$. In fact,

$$
\left\|x_{\alpha}^{\delta}-x^{\dagger}\right\| \leq c_{0} E \rho^{-1}\left(\frac{\delta}{E}\right)
$$

with $c_{0}=C+1+\frac{m}{C-1}$ for $1<C \leq 2$ and $c_{0}=C+1+m$ for $C \geq 2$.

Proof. Let us use notations (4.3). Due to $y^{\delta}-A x_{\alpha}^{\delta}=B_{\alpha}^{m} y^{\delta}$, the function $d$ in (4.1) can be written in the equivalent form $d(\alpha)=\left\|B_{\alpha}^{m+1} y^{\delta}\right\|$. Hence, for $\alpha$ chosen by rule (4.1) we have

$$
\left\|B_{\alpha}^{m+1} y\right\| \leq\left\|B_{\alpha}^{m+1} y^{\delta}\right\|+\left\|B_{\alpha}^{m+1}\left(y-y^{\delta}\right)\right\| \leq(C+1) \delta .
$$

From the concavity of $\varphi^{\frac{1}{m}}$ we obtain in analogy to the proof of (4.5) that

$$
g\left(r_{\alpha}^{2 m}(\lambda) \varphi^{2}(\lambda)\right) \leq \lambda^{2} r_{\alpha}^{2 m+2}(\lambda) \varphi^{2}(\lambda) .
$$

Exploiting (5.8) and (5.9), we obtain along the lines of the proof of Proposition 4.1 that for $\alpha$ chosen by rule (4.1) we have

$$
\left\|x_{\alpha}-x^{\dagger}\right\| \leq(C+1) E \rho^{-1}\left(\frac{\delta}{E}\right) .
$$

Now we follow the proof of Proposition 4.2 and obtain relation (4.8) in this case as well. Finally, we follow the proof of Theorem 4.3 and obtain the order optimal error bound (5.7) 


\section{Application}

In this section we consider a special inverse heat conduction problem which has been studied in [1]. This problem consists in identifying $w^{\dagger}=u\left(x_{1}, x_{2}, 0\right) \in$ $L^{2}\left(\mathbb{R}^{2}\right)$ from given data $y^{\delta}=u^{\delta}\left(x_{1}, x_{2}, 1\right) \in L^{2}\left(\mathbb{R}^{2}\right)$ where $y=u\left(x_{1}, x_{2}, 1\right)$ denotes the exact unperturbed data and $u\left(x_{1}, x_{2}, t\right)$ satisfies the heat equation

$$
u_{t}-\Delta u=0 \quad\left(\left(x_{1}, x_{2}, t\right) \in \mathbb{R}^{2} \times \mathbb{R}^{+}\right) .
$$

Let us transform the operator equation $A w=y, A \in \mathcal{L}(H)$ with $H=L^{2}\left(\mathbb{R}^{2}\right)$ into the frequency domain by means of the Fourier transform. Let $\widehat{w}\left(\xi_{1}, \xi_{2}\right)=$ $\mathcal{F}\left\{w\left(x_{1}, x_{2}\right)\right\}$ the Fourier transform of $w$, that is,

$$
\widehat{w}=\mathcal{F}\{w\}=\frac{1}{2 \pi} \int_{\mathbb{R}^{2}} w\left(x_{1}, x_{2}\right) \mathrm{e}^{-\mathrm{i}\left(x_{1} \xi_{1}+x_{2} \xi_{2}\right)} d x_{1} d x_{2}
$$

The inverse Fourier transform $w\left(x_{1}, x_{2}\right)=\mathcal{F}^{-1}\left\{\widehat{w}\left(\xi_{1}, \xi_{2}\right)\right\}$ is given by

$$
w=\mathcal{F}^{-1}\{\widehat{w}\}=\frac{1}{2 \pi} \int_{\mathbb{R}^{2}} \widehat{w}\left(\xi_{1}, \xi_{2}\right) \mathrm{e}^{\mathrm{i}\left(x_{1} \xi_{1}+x_{2} \xi_{2}\right)} d \xi_{1} d \xi_{2}
$$

Transforming the operator equation $A w=y$ into the frequency domain provides the equivalent operator equation $\widehat{A} \widehat{w}=\widehat{y}, \widehat{A} \in \mathcal{L}(H)$, in the form

$$
\mathrm{e}^{-\left(\xi_{1}^{2}+\xi_{2}^{2}\right)} \widehat{w}=\widehat{y}
$$

From this representation we realize that $A$ and $\widehat{A}$ are linear, non-negative, self-adjoint, injective and bounded operators with non-closed range where $\|A\|=\|\widehat{A}\|=1$.

In order to formulate our source condition we introduce the Sobolev scale $\left(H_{r}\right)_{r \in \mathbb{R}^{+}}$of positive real order $r$ (cf. [12]) according to $H_{0}=H=L^{2}\left(\mathbb{R}^{2}\right)$,

$$
H_{r}=\left\{w\left(x_{1}, x_{2}\right) \in H \mid\|w\|_{r}<\infty\right\}
$$

and norm

$$
\|w\|_{r}=\left(\int_{\mathbb{R}^{2}}\left(1+\xi_{1}^{2}+\xi_{2}^{2}\right)^{r}|\widehat{w}|^{2} d \xi_{1} d \xi_{2}\right)^{\frac{1}{2}}
$$

We impose as our source condition the smoothness condition $w^{\dagger} \in H_{p}$ for some $p>0$ with $\left\|w^{\dagger}\right\|_{p} \leq E$.

Now we are able to apply Theorem 2.1 and obtain 
Theorem 6.1. Let $M=\left\{w \in H_{p} \mid\|w\|_{p} \leq E\right\}$. If $\frac{\delta}{E} \leq 1$, then for the above formulated inverse heat conduction problem $A w=y$, or equivalently (6.1), the modulus of continuity of the inverse operator $A^{-1}$ on the source set $M$ is given by

$$
\omega(\delta, M)=E\left[1-\ln \frac{\delta}{E}\right]^{-\frac{p}{2}}(1+o(1)) \quad(\delta \rightarrow 0) .
$$

Proof. From (6.1) and (6.2) we conclude that in the frequency domain the source condition $w^{\dagger} \in M$ attains the equivalent form $\widehat{w}^{\dagger} \in M_{\varphi, E}$ with $M_{\varphi, E}$ given by

$$
M_{\varphi, E}=\left\{\widehat{w} \in H \mid \widehat{w}=\varphi(\widehat{A}) \widehat{v},\|\widehat{v}\|_{0} \leq E\right\}
$$

and $\varphi:(0,1] \rightarrow(0,1]$ given by

$$
\varphi(\lambda)=[1-\ln \lambda]^{-\frac{p}{2}} .
$$

This representation enables us to apply Theorem 2.1. Let us check Assumption A1 with $\varphi$ given by (6.4). It is easily seen that A1(i) is satisfied. Concerning A1(ii) we observe that the function $g$ of Assumption A1 is implicitly defined by $g\left([1-\ln \lambda]^{-p}\right)=\lambda^{2}[1-\ln \lambda]^{-p}$. From this representation we conclude that $g$ defined in Assumption A1(ii) is convex for $1-p \leq 2[1-\ln \lambda]$, which is true for $p>0$ and all $\lambda \in(0,1]$. Next, we have to compute $\rho^{-1}$ with $\rho$ given by (2.5). From (6.4) we obtain $\varphi^{-1}(\lambda)=\mathrm{e}^{1-\lambda^{-\frac{2}{p}}}$. Consequently, $\rho(\lambda):=\lambda \varphi^{-1}(\lambda)$ has the explicit form $\rho(\lambda)=\lambda \mathrm{e}^{1-\lambda^{-\frac{2}{p}}}$. This provides

$$
\rho^{-1}(\lambda)=[1-\ln \lambda]^{-\frac{p}{2}}(1+o(1)) \quad(\lambda \rightarrow 0) .
$$

Now we apply Theorem 2.1 and the proof is complete

Let us define the Lavrentiev regularized approximation in the frequency domain according to

$$
\widehat{w}_{\alpha}^{\delta}=\frac{\widehat{y}^{\delta}}{\mathrm{e}^{-\left(\xi_{1}^{2}+\xi_{2}^{2}\right)}+\alpha} .
$$

In order to apply Theorems 3.2 and 4.3 we have to check under which conditions the function $\varphi$ defined by (6.4) is concave. It can be easily realized that the inequality $\varphi^{\prime \prime}(\lambda) \leq 0$ is satisfied for $\lambda \leq \mathrm{e}^{-\frac{p}{2}}$. Hence, the function $\varphi$ is concave on the range $\left(0, \mathrm{e}^{-\frac{p}{2}}\right]$. Consequently, by scaling the operator $A$ by taking $\tilde{A}:=\mathrm{e}^{-\frac{p}{2}} A$ in place of $A$, so that $\|\tilde{A}\|=\mathrm{e}^{-\frac{p}{2}}$, we obtain for the Lavrentiev regularized approximation (6.5) the order optimal logarithmic type error bound

$$
\left\|\widehat{w}_{\alpha}^{\delta}-\widehat{w}^{\dagger}\right\|_{L^{2}\left(\mathbb{R}^{2}\right)}=O\left(\left[\ln \frac{1}{\delta}\right]^{-\frac{p}{2}}\right) \quad(\delta \rightarrow 0)
$$


provided $w^{\dagger} \in H_{p}$ and the regularization parameter $\alpha$ is either chosen a priori from the equation $\alpha \varphi(\alpha)=\frac{\delta}{E}$ with $\varphi$ according to (6.4) or a posteriori by rule (4.1).

Due to Plancherel's theorem we have $\|\widehat{w}\|=\|w\|$. Hence, the error bound (6.6) holds also true for the Lavrentiev regularized approximation $w_{\alpha}^{\delta}=\mathcal{F}^{-1}\left\{\widehat{w}_{\alpha}^{\delta}\right\}$ in the original domain, that is,

$$
\left\|w_{\alpha}^{\delta}-w^{\dagger}\right\|_{L^{2}\left(\mathbb{R}^{2}\right)}=O\left(\left[\ln \frac{1}{\delta}\right]^{-\frac{p}{2}}\right) \quad(\delta \rightarrow 0) .
$$

\section{Concluding remarks}

We have considered a priori and a posteriori parameter choice strategies for choosing the regularization parameter $\alpha$ involved in the method of Lavrentiev regularization and obtained order optimal error estimates under general source conditions.

It is apparent from equations (1.4) and (1.5) that if $A$ is a positive selfadjoint operator, then the method of Lavrentiev regularization is much simpler and more natural than the method of Tikhonov regularization. Not only that, the regularized equation (1.5) is in a form which can be adopted for its generalization to operator equations (1.1) on Banach spaces (see, e.g., [19, 23, 26]). Moreover, it is known that the method of Lavrentiev regularization is better suited than the method of Tikhonov regularization concerning speed of convergence and condition numbers in the case of finite dimensional approximation (see [26]). In spite of its simplicity, Morozov's discrepancy principle cannot be applied to it to yield convergence (see [7, 30]). Thus, for the method of Lavrentiev regularization it became necessary to adopt not-so-simple parameter choice strategies, as has been done in $[3-8]$. In contrast to the methods in $[3-8]$, the parameter choice strategy considered in this paper found appropriate to yield order optimality results under general source conditions which are applicable for a wide variety of situations.

Although optimality and order optimality results are available for the method of Tikhonov regularization under general source conditions, this work seems to be the first attempt for such consideration for the method of Lavrentiev regularization.

A natural generalization of the results of this paper would be to prove them for more general regularization methods which are defined by a general regularization scheme $x_{\alpha}^{\delta}:=g_{\alpha}(A) y^{\delta}$ with some properly chosen family of functions $\left\{g_{\alpha}: \alpha>0\right\}$ defined on $[0,\|A\|]$. The analysis for this generalization seems to be more complicated. We may attempt this in a future work.

Acknowledgement. The authors are grateful for the valuable hints of the anonymous referees that led to the improved version of the paper. 


\section{References}

[1] Ang, D. D., Gorenflo, R., Le, V. K. and D. D. Trong: Moment Theory and Some Inverse Problems in Potential Theory and Heat Conduction. Lect. Notes Math. 1792 (2002).

[2] Engl, H. W., Hanke, M. and A. Neubauer: Regularization of Inverse Problems. Dordrecht: Kluwer 2003.

[3] George, S. and M. T. Nair: An a posteriori parameter choice for simplified regularization of ill-posed problems. Int. Equ. \& Oper. Theory 16 (1993), 392 -399 .

[4] George, S. and M. T. Nair: A class of discrepancy principles for the simplified regularization of ill-posed problems. J. Austral. Math. Soc. (Series B) 36 (1994), $242-248$.

[5] George, S. and M. T. Nair: Error bounds and parameter choice strategies for simplified regularization in Hilbert scales. Int. Equ. \& Oper. Theory 29 (1997), $239-242$.

[6] George, S. and M. T. Nair: An optimal order yielding discrepancy principle for simplified regularization of ill-posed problems in Hilbert scales. Intern. J. Math. \& Math. Sci. 39 (2003), 2487 - 2499.

[7] Groetsch, C. W. and J. Guacaneme: Arcangeli's method for Fredholm equations of the first kind. Proc. Amer. Math. Soc. 99 (1987), 256 - 260.

[8] Guacaneme, J.: A parameter choice for simplified regularization. Rostocker Math. Kolloq. (Germany) 42 (1990), 59 - 68.

[9] Hofmann, B.: Regularization of Applied Inverse and Ill-Posed Problems. Leipzig: Teubner 1986.

[10] Hohage, T.: Regularization of exponentially ill-posed problems. Numer. Funct. Anal. \& Optimiz. 21 (2000), 439 - 464.

[11] Ivanov, V. and T. Korolyuk: Error estimates for solutions of ill-posed problems. USSR Comput. Math. Math. Phys. 9 (1969), 35 - 49.

[12] Krein, S. and Y. I. Petunin: Scales of Banach spaces. Russian Math. Surveys 21 (1966), $85-159$.

[13] Lavrentiev, M. M.: Some Improperly Posed Problems of Mathematical Physics. New York: Springer-Verlag 1967.

[14] Louis, A. K.: Inverse und schlecht gestellte Probleme. Stuttgart: Teubner 1989.

[15] Mair, B. A.: Tikhonov regularization for finitely and infinitely smoothing operators. SIAM J. Math. Anal. 25 (1994), 135 - 147.

[16] Mathé, P. and S. V. Pereverzev: Geometry of linear ill-posed problems in variable Hilbert scales. Inverse Problems 19 (2003), 789 - 803.

[17] Morozov, V. A.: On the solution of functional equations by the method of regularization. Soviet Math. Dokl. 7 (1966), $414-417$.

[18] Nair, M. T.: On Morozov's method for Tikhonov regularization as an optimal order yielding algorithm. Z. Anal. Anw. 18 (1999), 37 - 46. 
[19] Nair, M. T.: An iterated version of Lavretiev's method for ill-posed equations with approximately specified data. J. Inv. Ill-Posed Problems 8 (2000), 193 204.

[20] Nair, M. T.: Functional Analysis: A First Course. New Delhi: Prentice-Hall of India 2002.

[21] Nair, M. T., Schock, E. and U. Tautenhahn: Morozov's discrepancy principle under general source conditions. Z. Anal. Anw. 22 (2003), 199 - 214.

[22] Pereverzev, S. and E. Schock: Morozov's discrepancy principle for Tikhonov regularization of severely ill-posed problems. Numer. Funct. Anal. \& Optimiz. 21 (2000), $901-920$.

[23] Plato, R.: Resolvent estimates for Abel integral operators and the regularization of associated first kind integral equations. J. Int. Equ. 9 (1997), $253-278$.

[24] Raus, T.: Residue principle for ill-posed problems (in Russian). Acta Comment Univ. Tartuensis 672 (1984), $16-26$.

[25] Schock, E.: Approximate solution of ill-posed equations: arbitrary slow convergence vs. superconvergence. In: Constructive Methods for the Practical Treatment of Integral Equations (eds.: G. Hämmerlin and K. H. Hofmann). Basel: Birkhäuser Verlag 1985, pp. $234-243$.

[26] Schock, E.: Ritz-regularization versus least-squares-regularization. Solution methods for integral equations of the first kind. Z. Anal. Anw. 4 (1985), $277-284$.

[27] Tautenhahn, U.: Optimality for ill-posed problems under general source conditions. Numer. Funct. Anal. \& Optimiz. 19 (1998), 377 - 398.

[28] Tautenhahn, U.: On the method of Lavrentiev regularization for nonlinear illposed problems. Inverse Problems 18 (2002), 191 - 207.

[29] Tikhonov, A. N. and V. Y. Arsenin: Solution of Ill-Posed Problems. New York: Wiley 1977.

[30] Vainikko, G. M. and A. Y. Veretennikov: Iteration Procedures in Ill-Posed Problems (in Russian). Moscow: Nauka 1986.

Received 27.08.2003 